\title{
MATERIAL PROPERTIES AND OPTICAL GUIDING IN InGaAs-GaAs STRAINED LAYER SUPERLATTICES-A BRIEF REVIEW
}

\author{
Pallab K. Bhattacharya, Utpal Das, F. Y. Juang, Yasunobu Nashimoto $\dagger$ and \\ SUNANDA DHAR \\ Solid State Electronics Laboratory, Department of Electrical Engineering and Computer \\ Science, The University of Michigan, Ann Arbor, MI 48109, U.S.A.
}

(Received 7 June 1985; in revised form 3 July 1985)

\begin{abstract}
Due to the absence of lattice-matching requirements, strained-layer superlattices offer a large tunability in bandgap and other material properties suitable for device applications. Encouraging progress has been made in the molecular-beam epitaxial and metalorganic-vapor-phase-epitaxial growth of strained-layer superlattices and in their characterization. These have been briefly reviewed here. Since a strained-layer superlattice allows the use of $\operatorname{In}_{x} \mathrm{Ga}_{1-x}$ As layers with $x$-values up to -1.0 , a large variation of the refractive index from that in GaAs occurs due to mismatch strain and alloying. This variation in refractive index has been calculated. The increase in refractive index can be used to form optical guides in the SLS and such guides with good vertical confinement is demonstrated. Preliminary measurements of the impact-ionization parameters and deep-level traps in these materials are also reported. $\alpha / \beta$ values close to and slightly greater than unity are measured. A single electron trap with thermal activation energy equal to $0.16 \mathrm{eV}$ is identified.
\end{abstract}

\section{INTRODUCTION}

It is clear that superlattices are a new class of materials with potential for novel and exciting device applications. Lattice-matching requirements between the substrate and growing layers restrict the semiconductors which can be used. More freedom for bandgap tailoring is available with strained-layer superlattices (SLS) [1,2]. The well or barrier region of such a superlattice is usually composed of a semiconductor which is mismatched with the substrate. The mismatch energy is accommodated by the strain, provided the thickness of the strained layer is within certain limits. Relaxation of the requirements of lattice matching of individual layers allows the accommodation of a larger range of semiconductor properties for device applications. Early work on strained-layer superlattices revealed that there were difficulties involved in the growth and fabrication of devices. However, the concept is too appealing to forego and it is clear that important developments are imminent. To set the stage, a brief review of the development of strained-layer heterojunctions and superlattices and their device applications is made. Much of the early work has been done by Matthews and Blakeslee [3] and Osbourn and co-workers $[1,2,4,5]$. Our own work is described next. We have demonstrated optical guiding in a strained-layer superlattice, taking advantage of the large perturba-

fOn leave from NEC Corporation, Kawasaki, Japan.

‡On leave from the Institute of Radio Physics and Electronics, University of Calcutta, 92 Acharya PC Ray Road, Calcutta 700009, India. tion in dielectric constant that can be affected by alloying and strain. The systems of interest are the In $G$ a $A s-G$ aAs, G a A $s P-G$ a As $(G$ a $P)$, $G$ a $A s \mathrm{~S} b-\mathrm{G}$ a $\mathrm{A} / \mathrm{G}$ a $\mathrm{Sb} / \mathrm{A} 1 \mathrm{G}$ a $\mathrm{A} s$, InAs $\mathrm{Sb}_{1-x}-\operatorname{InAs} \mathrm{Sb}_{1-y}$, and GaAsP-InGaAs, although the growth and properties of the first two have been more intensively studied. The relation between bandgap and lattice constant for some of these binary and ternary systems is depicted in Fig. 1.

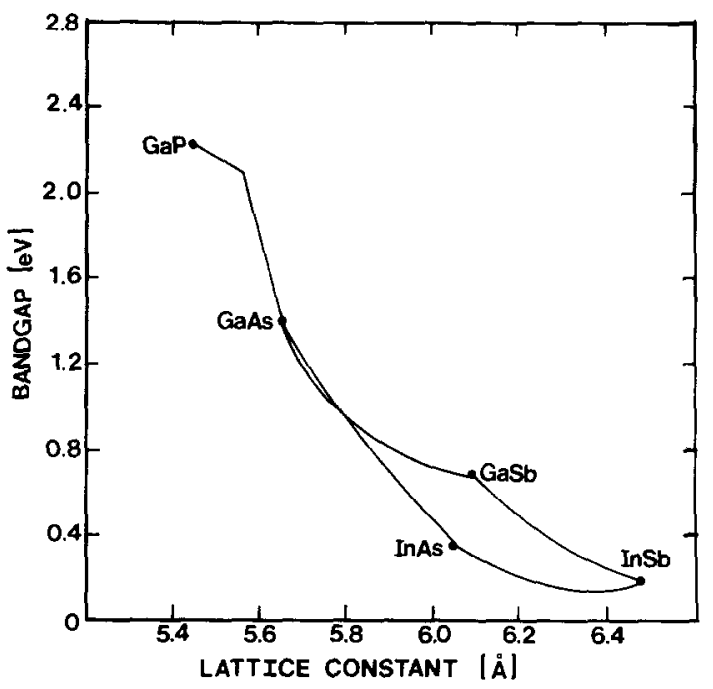

Fig. 1. Variation of bandgap with lattice constant in binary III-V semiconductors and the strained-layer ternary systems formed by their solid solutions. 


\section{PHYSICAL PRINCIPLES}

In the case of epitaxy of strained layers there exists a mismatch between the atoms on either side of the interface. The misfit $f_{x, y}$ is expressed as:

$$
f_{x, y}=\left(a_{2 x, y}-a_{1, x, y}\right) / \frac{1}{2}\left(a_{1 x, y}+a_{2 x, y}\right)
$$

where the subscripts 1,2 denote the epitaxial layer and the substrate, respectively, and $x, y$ refer to two suitably chosen interfacial directions. Compared to the unrelaxed state the energy of this bicrystal system can be reduced by two mechanisms: (a) generation of interfacial dislocations, and (b) compression or expansion which reduces the difference between the lattice parameters. For work on strained layers, it is the second mechanism which is of interest.

Defect-free growth is possible when the parabolic strain energy is less than the interfacial energy [6]. Based on the theories of van der Merwe[6] and Cabrera[7], Jesser and Kuhlmann-Wilsdorf [8] have shown, with some approximations, that dislocationfree growth can be obtained for thicknesses less than a critical thickness,

$$
h_{c}=-\frac{G_{2} b^{2} \ln \left[\frac{4 \pi G_{1} G_{2}\left(a_{2}-a_{1}\right)}{\left(G_{1}+G_{2}\right) G(1-\gamma)\left(a_{1}+a_{2}\right)}\right]}{2 \pi a_{2} f(1+2 \gamma)\left(G_{1}+G_{2}\right)}
$$

on an intinite substrate. Here $G_{1,2}$ are the shear moduli of the two materials, $\gamma \simeq \gamma_{1} \simeq \gamma_{2}$ is the approximate Poisson's ratio, $b=2 a_{1} a_{2} /\left(a_{1}+a_{2}\right)$ is the periodicity of the reference lattice and $G$ is the shear modulus of the reference lattice. The critical thickness given by eqn (2) is true for an infinite substrate and would actually represent a lower limit when the strain is shared by a substrate of finite thickness, as in a strained-layer superlattice.

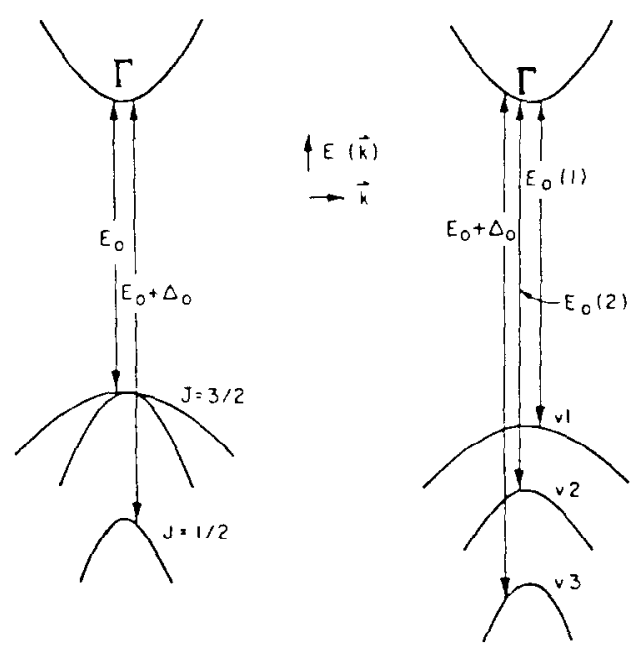

Fig. 2. Upper valence bands and the lowest conduction band in zincblende crystals in the absence (left) and presence (right) of uniaxial stress.
Matthews and Blakeslce[3] obscrved critical thicknesses higher than those calculated theoretically and attributed the difference to diffusion effects.

An immediate effect of the accommodation of mismatch strain is to change the bandgap of the materials. The roles of hydrostatic and shear strain in shifting the bands have been widely studied by piezobirefringence techniques in several semiconductors. Changes in the conduction and valence bands at $\vec{k}=0$ due to strain effects are depicted in Fig. 2. In going from a single heterostructure to a superlattice, the effects of quantization have to be taken into account.

Changes in the $(E-\vec{k})$ relation caused by strain result in changes of the carrier masses and dielectric constants. The relative dielectric constant in strained layers can be expressed as [9]

$$
\begin{aligned}
\epsilon_{r}(\omega)= & \frac{A}{2}\left\{\left(\frac{E_{o}}{E_{o}+\Delta E_{o 1}}\right)^{3 / 2} f\left(\chi_{1}\right)\right. \\
& +\left(\frac{E_{o}}{E_{o}+\Delta E_{o 2}}\right)^{3 / 2} f\left(\chi_{2}\right) \\
& \left.+\left(\frac{E_{o}}{\left(E_{o}+\Delta_{o}\right)+\Delta\left(E_{o}+\Delta_{o}\right)}\right)^{3 / 2} f\left(\chi_{s, 1}\right)\right\} \\
& +B
\end{aligned}
$$

Where $A$ and $B$ are material constants, $f(\chi)=$

$$
\begin{gathered}
\chi^{-2}\left[2-(1+\chi)^{1 / 2}-(1-\chi)^{1 / 2}\right], \chi=\hbar \omega / E_{i}, \\
E_{i}=E_{o}+\Delta E_{o l, 2}
\end{gathered}
$$

and

$$
\chi_{s o}=h \omega /\left[\left(E_{0}+\Delta_{0}\right)+\Delta\left(E_{0}+\Delta_{0}\right)\right] .
$$

\section{GROWTH OF STRAINED LAYER} SUPERLATTICE

Strained multilayers of GaAsP/GaAs were first realized by vapor-phase epitaxy[10]. At the present time strained-layer superlattices consisting of In$\mathrm{GaAs}-\mathrm{Ga \Lambda s}$ and GaAsP GaAs(GaP) are being grown by metal-organic-chemical-vapor deposition (MOCVD) and molecular-beam epitaxy (MBE). Other SLS systems are also being grown[11-15] for various device applications. In particular InAs-GaAs thin-layer SLS [15] on InP substrates may have important applications. We will briefly describe the growth of InGaAs-GaAs SLS being done in our laboratories by MBE. The GaAs substrates are prepared for growth by solvent degreasing and etching in $\mathrm{H}_{2} \mathrm{SO}_{4}: \mathrm{H}_{2} \mathrm{O}: \mathrm{H}_{2} \mathrm{O}_{2}(5: 1: 1)$. After initial desorption of oxides from the surface, growth of the superlattice is usually done at $520^{\circ} \mathrm{C}$ with an As pressure of $2 \times 10^{-5}$ Torr. The Ga flux rate corresponds to a GaAs growth rate of $1.8 \mu \mathrm{m} / \mathrm{hr}$ and the In flux is varied according to the alloy composition desired. 
There is little or no Indium re-evaporation at the growth temperature used. Assuming unity sticking coefficients for In and $\mathrm{Ga}$, the In concentration in the $\operatorname{In}_{x} \mathrm{Ga}_{1-x}$ As wells is approximately given by [16]:

$$
x=\frac{F_{\mathrm{In}}}{F_{\mathrm{Ga}}+F_{\mathrm{In}}}=1-\frac{n_{1} t_{2}}{n_{2} t_{1}},
$$

where $t_{1}$ and $t_{2}$ are the growth times of the GaAs and $\operatorname{In}_{x} \mathrm{Ga}_{1-x}$ As layers, respectivcly, and $n_{1}$ and $n_{2}$ are the number of GaAs and $\operatorname{In}_{x} \mathrm{Ga}_{1-x}$ As molecular layers in the superlattice. A typical SLS structure is schematically shown in Fig. 3. After the growth of a $0.3 \mu \mathrm{m}$ GaAs buffer layer, an intermediate ternary buffer layer, with $x$ having a value between 0 and the $x$-value of the ternary layer in the superlattice, is grown. This is followed by the growth of the superlattice. In a SLS structure the lattice mismatch is accommodated by alternate expansion and compression in the plane of the layer resulting in a twodimensional lattice strain. The tetragonal expansion and compression of the lattice of successive layers has been directly observed by high resolution electron microscopy [17] and by ion-channeling studies [18].

An insight into the growth mechanism of strained layers can be obtained by qualitatively noting the in situ electron diffraction (RHEED) pattern of the growing surface or by quantitatively monitoring the intensity of the specular beam. We have observed that after some monolayers of InGaAs growth on GaAs, dependent on the InAs content, the original streaked RHEED pattern of a two-dimensional surface is less resolved and changes to a slightly spotty pattern. This indicates that the mismatchinduced strain causes a rearrangement of the atoms of the growing film. Similar observations have been reported by Lewis et al. [19].

$\mathrm{X}$-ray diffraction patterns of SLS structures can give important information regarding their structural properties. Analysis of X-ray diffraction data is currently being done by us and other workers[20]. Fig-

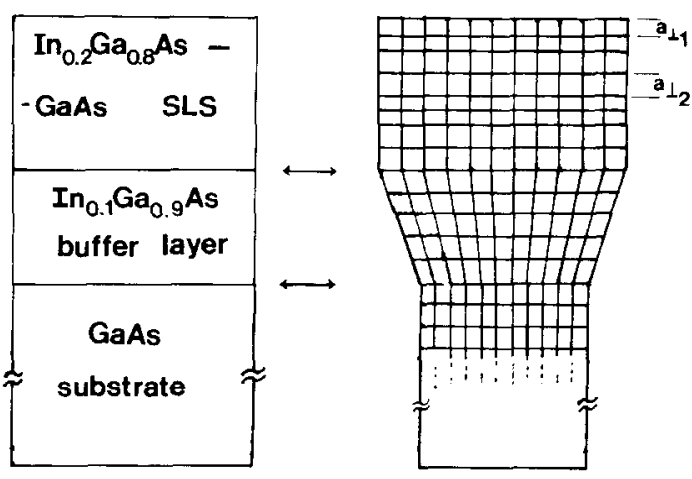

Fig. 3. Schematic of SLS structure grown by molecular beam epitaxy and the alternate layers within the superlattice in compression and tension.

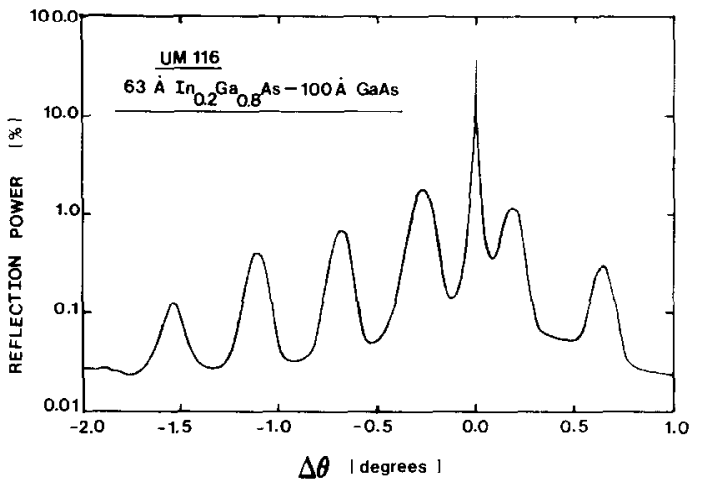

Fig. 4. Measured X-ray rocking curve in a $\mathrm{In}_{0.2} \mathrm{Ga}_{0.8}$ As-GaAs SLS with $\mathrm{FeK}_{\alpha \mathrm{i}}$ (400) reflection.

ure 4 shows the $X$-ray diffraction pattern obtained for one of our samples using a double-crystal X-ray diffractometer. The superlattice consists of periods of $100 \AA$ GaAs barriers and $63 \AA \mathrm{In}_{0.2} \mathrm{Ga}_{0.8}$ As wells, wherein the lattice mismatch is $-1.4 \%$. The periodic structure results from the superlattice. The angle $\Delta \theta$ is plotted relative to the Bragg angle of the GaAs substrate peak. Using a kinematic model for the diffraction process, it is possible to deduce the average layer thickness and variations in the thickness, their parallel and perpendicular strains and the composition of the InGaAs layers. These results will be reported elsewhere.

An obvious and important question in relation to device applications is the structural integrity of the SLS and the effects of doping, implantation and diffusion on them. It has been demonstrated [21] that doped layers and $p-n$ junctions can be made in the SLS during growth. Camras et al.[22] have shown that $\mathrm{Zn}$ diffusion in a $\mathrm{GaP}-\mathrm{GaAs}_{1-x} \mathrm{P}_{x}$ superlattice enhances the interdiffusion of $A$ s and $P$ atoms at the heterointerfaces resulting in a disordered $\mathrm{GaAs}_{1-,} \mathrm{P}_{\text {. }}$ $(y \neq x)$ alloy. Arnold et al. [23] have demonstrated from Rutherford backscattering spectrometry and cantilever beam techniques that composition modulations of the SLS are maintained even for ion fluences corresponding to deposited energy densities of $\sim 10^{20} \mathrm{KeV} / \mathrm{cm}^{3}$ into collisional processes.

\section{ELECTRICAL AND OPTICAL PROPERTIES ANI) DEVICE APPLICATIONS}

It is evident from what has been discussed in the previous section that SLS with good structural and compositional integrity can be grown by $\mathrm{MBE}$ or MOCVD. For device applications, their electronic and optical properties are of equal importance. Photoluminescence and absorption measurements on SLS structures have been made by us and other authors [24-27]. The intensity of the exciton related transition is dependent on the quality of the SLS and the growth technique used, but has been shown to be higher than that of single layer MOCVD GaAs [24]. A typical low-temperature photoluminescence spec- 
trum measured by us for a $\operatorname{In}_{0.2} \mathrm{Ga}_{0.8}$ As $(63 \AA)-\mathrm{GaAs}$ (50 A) SLS is shown in Fig. 5. The spectrum is dominated by a strong peak at $1.308 \mathrm{eV}$ which is attributed to a $n=1$ exciton transition between an electron and a $|3 / 2,3 / 2\rangle$ heavy hole. The transition energy is confirmed by results from an approximate Kronig-Penney calculation. The FWHM of the transition lineshape is $\sim 6 \mathrm{meV}$ and is one of the narrowest linewidths that has been observed for $63 \AA$ wells. The variation of the superlattice bandgap with temperature follows the law

$$
E_{g}(T)=E_{o}-\alpha T^{2}
$$

and the values of $E_{o}$ and $\alpha$ have been determined by Anderson et al [28] from temperature-dependent photoluminescence spectra.

Electron mobilities in both uniformly doped and modulation doped $\operatorname{In}_{0.2} \mathrm{Ga}_{0.8} \mathrm{As}-\mathrm{GaAs}$ [28] and modulation doped $\mathrm{GaAs}_{1-x} \mathrm{P}_{x}-\mathrm{GaAs}$ [29] and $\mathrm{GaAs}_{1-x} \mathrm{Sb}_{x}-\mathrm{Al}_{y} \mathrm{Ga}_{1-\gamma}$ As[14] SLS have been measured. Low temperature mobility in the 2DEG of the modulation doped structures comparable to those in AlGaAs-GaAs superlattices has been measured. The results confirm that high crystalline quality in the SLS can be obtained in spite of the large mismatch. A measure of the carrier transport properties in a SLS is also obtained from the minority carrier lifetimes. These have been recently measured in both $n$ and $p$-type $\mathrm{GaAs}_{x} \mathrm{P}_{1-x}-\mathrm{GaP}$ SLS by Gourley et al. [30]. In both types of materials $L_{\|}-1.5 \mu \mathrm{m}$ and $L_{\perp} \sim 0.15 \mu \mathrm{m}$. The latter value suggests that thermionic emission is the dominant mode of transport normal to the layers and also indicates that the material is suitable for emitter and detector applications.

Deep level traps in GaAsP-GaP SLS have been investigated by Barnes et al. [31]. These authors report that the number of trap centers and their concentrations are low and the trap depth profiles are more uniform than that in the alloyed buffer layer

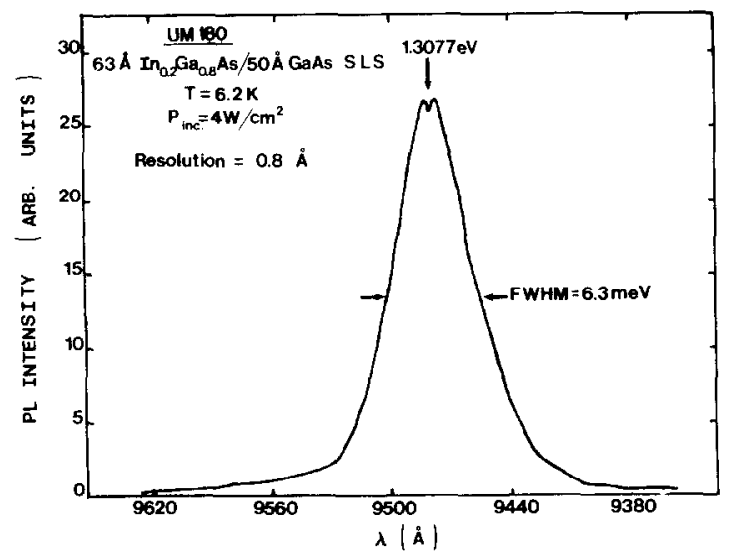

Fig. 5. Bound exciton transition observed in the low-temperature photoluminescence spectrum of a In $_{0.2} \mathrm{Ga}_{0.8} \mathrm{As}-\mathrm{GaAs}$ SLS grown by MBE.

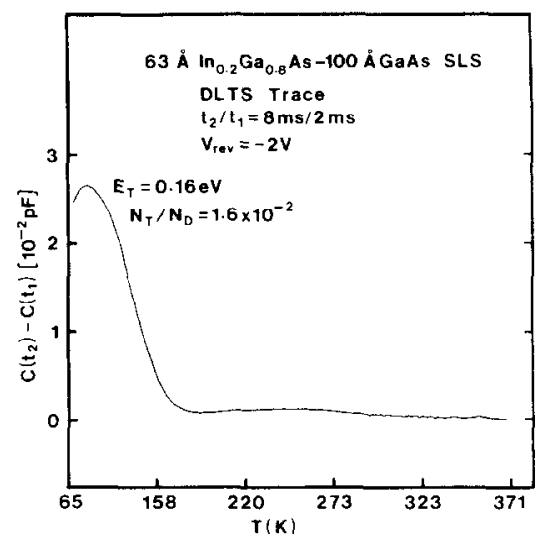

Fig. 6. Deep level transient spectroscopy data obtained from measurements on diode formed with $\operatorname{In}_{0.2} \mathrm{Ga}_{0.8}$ As-GaAs SLS. The single positive-going peak at low-temperature corresponds to an electron-trap activation energy of $0.16 \mathrm{eV}$. The free electron concentration in the sample, obtained form $C-V$ measurements, is $3.0 \times 10^{16}$ cill $^{-3}$.

between the substrate and the SLS. We have, for the first time, investigated deep level traps in MBE-grown $\mathrm{In}_{0.2} \mathrm{Ga}_{0.8} \mathrm{As}-\mathrm{GaAs}$ SLS using deep level transient spectroscopy on $p^{+}-n$ SLS mesa diodes. The results are shown in Fig. 6. A single electron trap with thermal activation energy equal to $0.16 \mathrm{eV}$ and small concentration is detected. Reverse bias-dependent measurements indicate that the concentration increases with distance from the graded buffer layer. A hole trap with small concentration might be present. Further work is in progress. Generally, recombination at heterostructure interfaces occurs at misfit locations, if present. Studies on recombination centers in strained heterojunctions have been made by Ettenberg and Olsen [32] and Aspnes [33]. It was concluded that it is desirable to have each successive layer with a smaller lattice parameter than the one preceding it. The interface recombination velocity in In $\mathrm{Ga}_{1-x} \mathrm{P}-\mathrm{GaAs}$ heterojunctions is linearly related to the lattice parameter mismatch and the data can be fitted with capture cross-section $\sigma \sim 10^{-14}-10^{-15}$ $\mathrm{cm}^{2}$.

Structural integrity, large bandgap tunability and desirable electrical and optical properties in SLS have led to the fabrication and demonstration of a large number of devices $[11,13,34-38]$. We will not, however, go into a detailed description of the performance of thesc devices.

\section{REFRACTIVE INDEX AND STRAIN-INDUCED OPTICAL GUIDING}

Strain induced perturbation of the refractive index is a uscful property for the fabrication of optical waveguides [39]. Calculations for asymmetric waveguides[40] show that a refractive index step of $\sim 0.001$ is necessary for optical confinement at a wavelength of $-0.89 \mu \mathrm{m}$ with a guide thickness of 3 $\mu \mathrm{m}$. However, for higher wavelengths and smaller 
thicknesses the refractive index step necessary is at least one order higher. This is not easily achieved with a striped geometry structure. Strained In $\mathrm{Ga}_{1-x}$ As on GaAs are potentially excellent materials for this purpose as InAs has the highest refractive index amongst the common III-V binary semiconductors. It is evident, however, that any suitable thickness of a single In $\mathrm{Ga}_{1-x}$ As strained layer on a GaAs substrate for use in guiding or modulation cannot be grown. This is made possible by a SLS, where higher $x$-values in the well regions can be used. In fact, the change of refractive index due to alloying is larger than that due to mismatch strain.

Starting from the Kramers-Kronig integral dispersion relations, we have recently calculated the strain and refractive index change in the ternary layer of In $\mathrm{Ga}_{1 \ldots x}$ As-GaAs heterojunctions [41]. In addition, the refractive index variation with mismatch strain and with alloying in the ternary layer has been explicitly determined. The strain in the ternary layers is calculated using

$$
\epsilon-\left(S_{11}+S_{12}\right) \sigma
$$

where $\sigma$ is the compressive biaxial stress and $S_{i j}$ is the compliance constants of the material. In the region close to but below the bandgap, the refractive index $n(\omega)$ can be expressed by

$$
n(\omega)=\left[\epsilon_{r}(\omega)\right]^{1 / 2}
$$

where the dielectric constant $\epsilon_{r}$ is calculated from eqn (3). Details of the calculation are described in [41].

Figure 7 depicts the calculated variation of strain in the ternary layer as a function of $x$ for different thicknesses of InGaAs and GaAs layers. The strain in the GaAs layer of finite thickness has also been taken into account. The variation of refractive index,

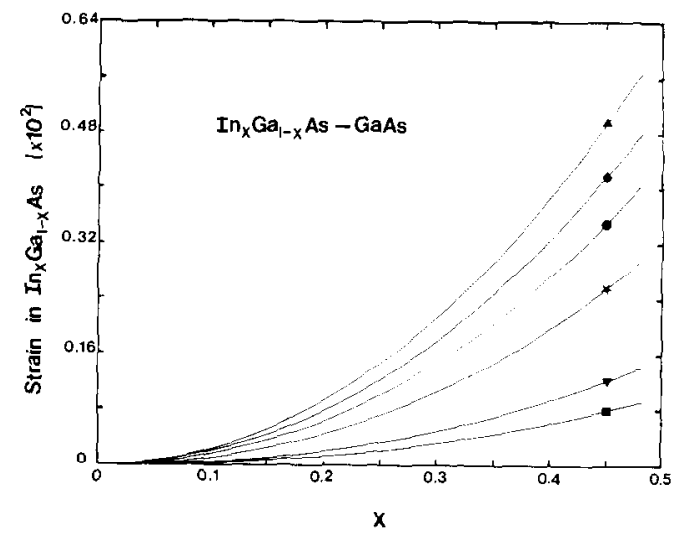

Fig. 7. Strain in the $\operatorname{In}_{x} \mathrm{Ga}_{1-x}$ As layer of a InGaAs-GaAs SLS for the following combination of thicknesses indicated by representative symbols: $(\boldsymbol{\Lambda})$ (InGa)As $=25 \AA$ A, $\mathrm{GaAs}=70$

$\AA:(\bullet)(\operatorname{InGa}) \mathrm{As}=50 \AA, \mathrm{GaAs}=100 \AA ;(*)(\mathrm{InGa}) \mathrm{As}=70$

$\AA$, GaAs $=70 \AA$; (v) $(\operatorname{InGa}) A s=100 \AA ., G a A s=50 \AA$;

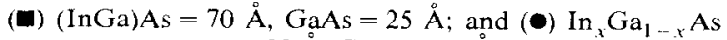
$=30 \AA, \mathrm{GaAs}=45 \AA$

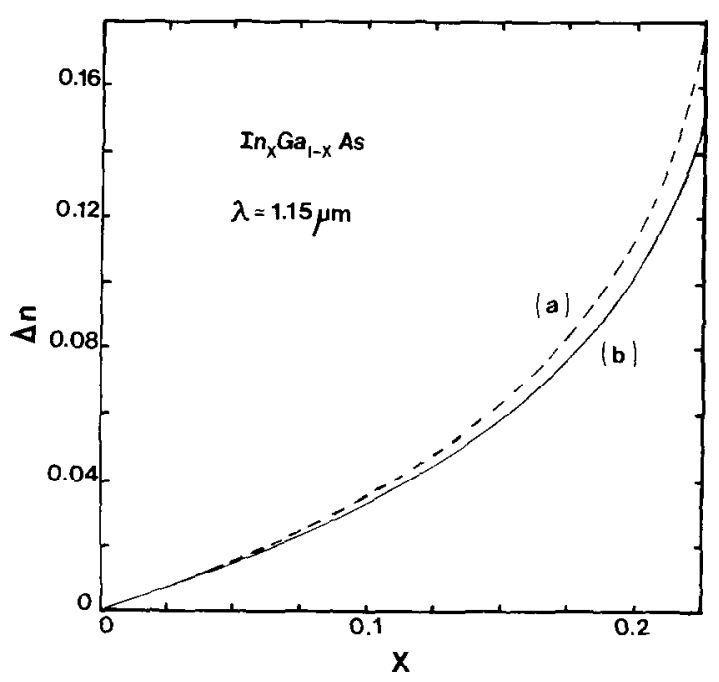

Fig. 8. Variation of refractive index with composition in (a) a strain-free layer of $\operatorname{In}_{x} \mathrm{Ga}_{1-x}$ As and (b) in the same layer grown with mismatch induced strain on a GaAs substrate. In case (b) it is assumed that the thickness of the ternary layer is less than the critical value above which dislocations are induced.

compared to that in $\mathrm{GaAs}$, in a single $\ln _{x} \mathrm{Ga}_{1}{ }_{x}$ As layer is shown in Fig. 8. Two cases have been considered. In the first it is assumed that the ternary layer is strain-free and in the second the effect of mismatch with a GaAs substrate (infinite thickness) is considered. It is seen that strain causes an additional decrease of refractive index, though the effect is small compared with that for alloying. The data presented in Figs. 7 and 8 are for single heterojunctions, whereas the usefulness of ternary layers with large $x$, giving a large refractive index change, can only be realized in a superlattice structure. Therefore, quantization effects need to be included to determine the true refractive index of the superlattice.

We have grown $2 \mu \mathrm{m} \operatorname{In}_{0.2} \mathrm{Ga}_{0.8}$ As-GaAs SLS structures by $\mathrm{MBE}$ to investigate their optical guiding properties. Single and coupled channel waveguides are fabricated by forming thin gold stripes on the SLS surface. Guide modes were excited by focusing a suitably shaped and focussed $1.15 \mu \mathrm{m}$ laser beam onto a waveguide cross-section exposed by cleaving. Extremely strong and well defined vertical confinement is observed in the SLS. Such confinement is usually not seen in GaAs without applying a bias (electro-optic) or external strain. It is confirmed that refractive index change caused by mismatch strain and alloying in the SLS is the dominant guiding mechanism. The photomicrograph of a guided mode is shown in Fig. 9. This is, to our knowledge, the first demonstration of index-guiding in SLS structures. The work is in a preliminary stage and more advanced structures are being fabricated. It is clear, however, that SLS would be useful materials for passive optical components to be coupled with active devices in an optically integrated circuit. 


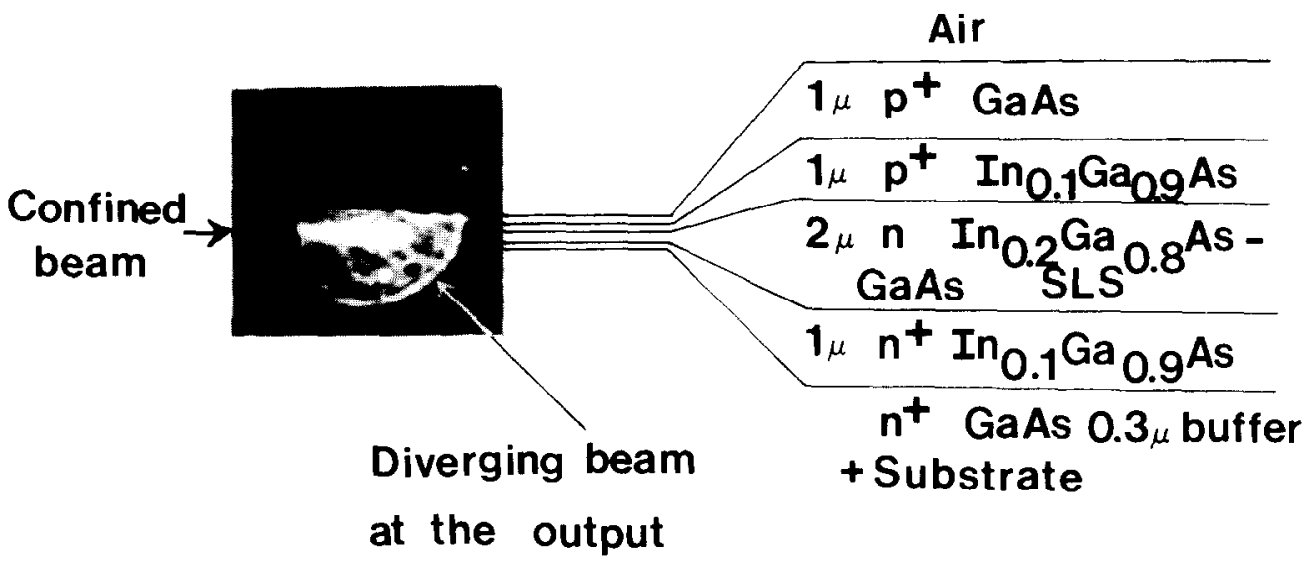

Fig. 9. Light output from a $4 \mathrm{~mm}$ long $\mathrm{In}_{0.2} \mathrm{Ga}_{0.8} \mathrm{As}-\mathrm{GaAs}$ index guide. The $1.15 \mu \mathrm{m}$ focused incident light remains confined in the SLS.

\section{ELECTRON AND HOLE IMPACT IONIZATION COEFFICIENTS}

Enhanced values of the impact ionization coefficient ratio are desirable for low-noise avalanche photodiodes and for microwave devices such as the IMPATT. It is therefore of interest to measure the impact ionization coefficients in strained layer superlattices. Preliminary data of such measurements in $\mathrm{In}_{0.2} \mathrm{Ga}_{0.8}$ As-GaAs SLS avalanching regions are presented here.

Mesa etched $p^{+}-n-n^{+}$diodes were fabricated from the MBE-grown SLS structures. Pure electron and hole injection was achieved by focusing a $5 \mu \mathrm{m}$ diameter $6328 \AA$ He-Ne laser beam onto the $p^{+}$or the $n^{+}$region, respectively, of the reverse-biased diode. The multiplication coefficients were measured as a function of bias. From the measured values of the electron and hole multiplication factors the impact ionization coefficients were derived by assuming a bulk-like nature of SLS as far as avalanche multiplication is concerned. Measured values of $\alpha\left(E_{m}\right)$ and $\beta\left(E_{m}\right)$ in a $2 \mu \mathrm{m} \operatorname{In}_{0.2} \mathrm{Ga}_{0.8}$ As $(63 \AA)-\mathrm{GaAs}$ $(100 \AA)$ are plotted in Fig. 10. This is probably the first report on $\alpha$ and $\beta$ in strained layer super-

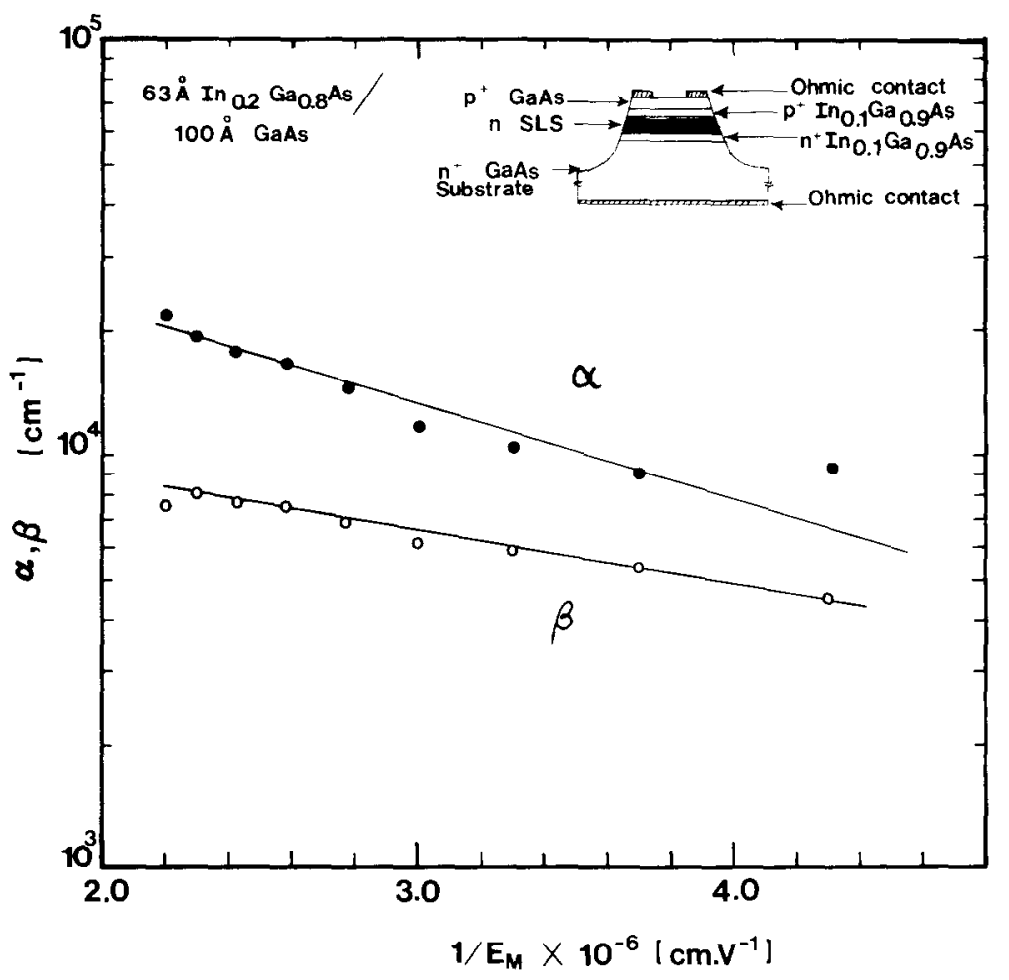

Fig. 10. Electron and hole impact ionization coefficients measured in a (63 A) $\ln _{0,2} \mathrm{Ga}_{6,8} \mathrm{As}-(100 \mathrm{~A})$ GaAs strained layer superlattice. 
lattices. More detailed measurements are in progress. It is clear, however, that non-equal values of $\alpha$ and $\beta$ can be obtained in these superlattices. Similar measurements are being made by Bulman et al. [42]. The measured values of $\alpha$ and $\beta$ suggest that impact ionization properties in the SLS are almost bulk-like. Slightly enhanced values of $\alpha / \beta$ at higher fields may be due to unequal scattering rates of electrons and holes in their respective quantum wells.

\section{CONCLUSIONS}

A brief review of the physical properties of strained materials, and the $\mathrm{MBE}$ growth and propertics of In $\mathrm{Ga}_{1-x}$ As-GaAs and other systems of strained layer superlattices has been made. The variation of refractive index with alloying and strain in single strained heterojunctions has been calculated. Based on the change in refractive index with alloy composition in $\mathrm{In}_{x} \mathrm{Ga}_{1-x}$ As it is evident that InGaAs-GaAs and other SLS systems should form efficient optical waveguides. Index guiding in a InGaAs-GaAs SLS has been observed for the first time and the results confirm theoretical predictions. Results from measurements of the impact ionization coefficients are also reported. $\alpha / \beta \sim 1.4-2.5$ are measured in the bias range used. DLTS measurements reveal one dominant electron trap with an activation energy of $0.16 \mathrm{eV}$. The flexibility in the choice of materials provided by SLS and their attractive material properties enhances their importance for future device technology. At the same time, more studies are needed to ascertain their structural behavior and extend ongoing measurements to determine their specific heat, thermal expansion coefficient, and other important physical parameters.

Acknowledgements - The authors wish to thank Dr. B. Paine and Mr. K. S. Huang at California Institute of Technology for making the $\mathrm{X}$-ray diffraction measurements. The work is being supported by the National Aeronautical and Space Administration, Langley Research Center, under Grant NAG-1-555. Partial support by a grant from Instruments, S.A., RIBER division is also acknowledged.

\section{REFERENCES}

1. G. C. Osbourn, J. Appl. Phys. 53, 1586 (1982).

2. G. C. Osbourn, R. M. Biefeld and P. L. Gourley, Appl. Phys. Lett. 41, 172 (1982).

3. J. W. Matthews and A. E. Blakeslee, J. Crystal Growth 27, 18 (1974).

4. G. C. Osbourn, Phys. Rev. B27, 5126 (1983).

5. P. L. Gourley, R. M. Biefeld, G. C. Osbourn and I. J. Fritz, Inst. Phys. Conf. Ser. 65, 241 (1983).

6. J. H. van der Merwe, J. Appl. Phys. 34, 117 (1963).

7. N. Cabrera, Surf. Sci. 2, 320 (1964).

8. W. A. Jesser and D. Kuhlmann-Wilsdorf, Phys. Stat. Sol. 19, 95 (1967)

9. S. Adachi and K. Oe, J. Appl. Phys. 54, 6620 (1983).

10. J. W. Matthews and A. E. Blakeslee, J. Crystal Growth 32, 265 (1976), and references therein.
11. S. M. Bedair, T. Katsuyama, M. Timmons and M. A. Tischler, IEEE Electron. Dev. Lett. EDL-5, 45 (1984).

12. G. C. Osbourn, J. Vac. Sci. Technol. B2, 176 (1984).

13. J. Klem, R. Fischer, W. T. Masselink, W. Kopp and H. Morkoc, J. Appl. Phys. 55, 3843 (1984).

14. H. P. Wei, M. J. Chou, D. C. Tsui, J. Klem, H. Morkoc and R. J. Wagncr, Appl. Phys. Lett. 45, 1292 (1984).

15. M. C. Tamargo, R. Hull, L. H. Greene, J. R. Hayes, and A. Y. Cho, Appl. Phys. Lett. 46, 569 (1985).

16. M. Quillec, L. Goldstein, G. Le Roux, J. Burgeat and J. Primot, J. Appl. Phys. 55, 2904 (1984).

17. J. M. Brown, N. Holonyak, M. J. Ludowise, W. T. Dietze and C. R. Lewis, Appl. Phys. Lett. 43, 863 (1983).

18. S. T. Picraux, L. R. Dawson, G. C. Osbourn and W. K. Chu, Appl. Phys. Lett. 43, 930 (1983).

19. B. F. Lewis, T. C. Lee, F. J. Grunthaner, A. Madhukar, R. Fernandez and J. Maserjian, J. Vac. Sci. Technol. B2, 419 (1984).

20. W. D. Laidig, C. K. Peng and Y. F. Lin, J. Vac. Sci. Technol. B2, 181 (1984); also, R. M. Biefeld, G. C. Osbourn, P. L. Gourley and I. J. Fritz, J. Electron. Mater. 12, 903 (1983).

21. L. R. Dawson, G. C. Osbourn, T. E. Zipperian, J. J Wiczer, C. E. Barnes, I. J. Fritz, and R. M. Biefeld, $J$. Vac. Sci. Technol. B2, 179 (1984).

22. M. D. Camras, N. Holonyak, K. Hess, M. J. Ludowise, W. T. Dietze and C. R. Lewis, Appl. Phys. Lett. 42, 185 (1983).

23. G. W. Arnold, S. T. Picraux, P. S. Peercy, D. R. Myers and L. R. Dawson, Appl. Phys. Lett. 45, 382 (1984).

24. P. L. Gourley and R. M. Biefeld, J. Vac. Sci. Technol. 21, 473 (1982); also Appl. Phys. Lett. 45, 749 (1984).

25. J. Y. Marzin and E. V. K. Rao, Appl. Phys. Lett. 43, $560(1983)$.

26. H. Kato, M. Nakayama, S. Chika and N. Sano, Solid-St. Commun. 52, 559 (1984).

27. N. G. Anderson, W. D. Laidig and Y. F. Lin, $J$. Electron. Mater. 14, 187 (1985).

28. I. J. Fritz, I. R. Dawson and T. F. Zipperian, Appl Phvs. Lett. 43, 846 (1983)

29. M. Ludowise, W. T. Dietze, C. R. Lewis, P. Gavrilovic, T. C. Hsieh and K. Hess, I. Appl. Phvs. 54, 6771 (1983).

30. P. L. Gourley, R. M. Biefeld, T. E. Zipperian and J. J. Wiczer, Appl. Phys. Lett. 44, 983 (1984).

31. C. E. Barnes, R. M. Biefeld, T. E. Zipperian and G. C. Osbourn, Appl. Phys. Lett. 45, 408 (1984).

32. M. Ettenberg and G. H. Olsen, J. Appl. Phys, 48, 4275 (1977).

33. D. E. Aspnes, Surf. Sci. 132, 406 (1983).

34. M. J. Ludowise, W. T. Dietze, R. Boettcher and N. Kaminar, Appl. Phys. Lett. 43, 468 (1983).

35. M. J. Ludowise, W. T. Dietze, C. R. Lewis, M. D. Camras, N. Holonyak, B. K. Fuller and M. A. Nixon, Appl. Phys. Lett. 42, 487 (1983).

36. W. D. Laidig, Y. F. Lin and P. J. Caldwell, J. Appl. Phys. 57, 33 (1985).

37. D. R. Meyers, J. J. Wiczer, T. E. Zipperian and R. M. Biefeld, IEEE Electron. Dev. Lett. EDL-5, 326 (1984).

38. G. C. Osbourn, J. Vac. Sci. Technol. B2, 176 (1984).

39. P. A. Kirkby, P. R. Selway and L. D. Westbrook, $J$. Appl. Phys. 50, 4567 (1979).

40. R. G. Hunsperger, Integrated Optics: Theory and Technology, p. 37. Springer-Verlag, New York (1982).

41. U. Das and P. K. Bhattacharya, J. Appl. Phys. to be published.

42. G. E. Bulman, L. R. Dawson, J. J. Wiczer and T. E. Zipperian, 27th Annu. Electron. Mater. Conf., Boulder, CO 1985. 